\title{
37. On the Relationship between the Mode of Stimulation of Cortical Motor Area and Spinal Reflex Activity
}

\author{
Jiro Tatsuno and Utaka Matsumoto \\ Dept. of Neuropsychiatry, School of Medicine, Chiba University
}

The spacial and temporal summation of the EPSP's of the motoneuron of spinal cord evoked by the stimulation of motor cortex and peripheral afferent nerve of cats was already discussed at the previous meeting of the society, and then the suggestion was added that the summation was modified by the change of mode of central stimulation.

In this paper, we used the various frequencies and number of train of rectangular pulse for the cortical stimulation and observed its effect on the spinal reflex activity evoked by the stimulation of muscular nerve of $\mathrm{m}$. semitendinosus, recording the responses at the L7 ventral root of cats. When the number of the train of the cortical stimulation increased, the response time of the spinal reflex decreased. When the frequency of the stimulation increased the time course was shortened in the relation of the interval of stimulations and response time. From these observations we will refer to the mechanism of the special reflex.

38. Inhibitory and Facilitatory Effects upon the Splanchnic Evoked Potential on the Spinal Level as well as on the Thalamic Level

\author{
Miyoshi Urabe, Takashi Tsubokawa, Hiraki Sakurai, Satoru Kadoya, \\ Masao Seki and Hiro Watanabe \\ Dept. of Surgery, School of Medicine, University of Kanazawa
}

The evoked potentials to the stimulation of the splanchnic nerve were picked up in the cervical regions of the spinal cord and in the thalamic level of the unanesthetized animals. The effects on these evoked potentials were obsrved after the stimulation of the peripheral nerve or the central nervous system.

1) The splanchnic evoked potential in the anterolateral funiculus of the spinal cord was inhibited by the vagal stimulation $(5-20 / \mathrm{sec})$ and the inhibitory effect was remained even after the transection at the intracollicular level. Although the splanchnic evoked potential in the anterolateral funiculus was also inhibited by high frequency stimulation $(50-100 / \mathrm{sec})$, this inhibitory effect completely disappeared after the transection at the same level. 
2) The high frequency stimulation of the trigeminal or the sciatic nerve inhibited the splanchnic evoked potential in the anterolateral funiculus, however, the low frequency stimulation $(5-20 / \mathrm{sec})$ did not exhibit any change in the evoked potential.

3) The stimulation of the abdominal muscles as well as the dorsal muscles of the trunk inhibited the splanchnic evoked potential in the anterolateral funiculus. This effect was observed even in the spinal animal though the effect disappeared after the section at the intracollicular level in some cases.

4) The splanchnic evoked potentials were picked up not only in VPL but also in CM-Pf complex and the magnocellular part of the medial genicular body at the thalamic level.

5) In CM-Pf complex of the thalamic level, the splanchnic evoked potential was interacted by the cutaneous evoked potential, when the individual nerve was stimulated at an interval of $60-80 \mathrm{msec}$. In VPL, however, no interaction was seen between the both evoked potentials.

6) The splanchnic evoked potential in CM-Pf complex completely disappeared but the spontaneous activity in CM-Pf complex changed characteristically to the spindle bursts of $25-27 / \mathrm{sec}, 50 \mathrm{mV}$ during and after the tetanic stimulation of the splanchnic nerve. On the other hand, the splanchnic evoked potential and the spontaneous discharges, in VPL, did not show any change to the same stimulation.

39. Electrophysiologic Study on the Function of the Automatic Nervous System in the Movement of the Biliary Tract

\section{Seiji IKEDA}

Dept. of Surgery, Mie Prefectural University School of Medicine

Although a considerable amount of roentogenological study has been done on the autonomic innervation of the biliary tract, little is known about it. On the other hand, inquiries into the action current in the intestinal smooth muscles have been rapidly developed in a wide range with the advancement of physiological studies on the movement of the alimentary tract, however reports relating to the action current of the biliary tract are rare.

With dogs, action currents of the gallbladder, common duct, sphincter of Oddi and duodenum were observed electrophysiologically with the extracellular electrode method.

The results were as follows.

1) The action currents of the gallbladder and common duct in the normal 